\section{Ambulant erworbene Pneumonien stellen große Belastung dar}

Kolditz $\mathrm{M}$ et al. Burden and risk factors of ambulatory or hospitalized CAP: A population based cohort study. Respir Med 2016; 121: $32-38$

Ambulant erworbene Pneumonien (CAP) zeigen sehr variable Verläufe und Behandlungsergebnisse mit publizierten Mortalitätsraten von unter $1 \%$ bis über $40 \%$. Auch die Inzidenzdaten variieren deutlich und unterscheiden sich im Hinblick auf Land, Population und Studiendesign. Große populationsbasierte Datenanalysen zur CAP und deren Verlauf, die auch Risikofaktoren wie Alter oder Begleiterkrankungen beinhalten, sind rar, wären aber dringend nötig.

Ziel der vorliegenden Studie war es daher, Inzidenz, Hospitalisierungsrate und 30-Tages-Mortalität von ambulanten und hospitalisierten CAP-Patienten zu analysieren und diesbezügliche Risikofaktoren zu ermitteln. Hierzu nutzten die Autoren Daten einer großen deutschen Krankenkasse, die mehr als die Hälfte der erwachsenen Bevölkerung im Bundesstaat Sachsen abdeckt. Vergleiche mit Daten des statistischen Bundesamts zeigten, dass die erfassten Personen eine repräsentative Auswahl darstellten. Die Autoren erfassten Daten zur CAP über die entsprechenden ICD10 Codes, und zwar für die Jahre 20102011. Stationäre Fälle wurden über die Entlassdiagnosen erfasst, ambulante Fälle über die Diagnosecodes ohne eine Hospitalisierung aufgrund einer CAP innerhalb von 30 Tagen, validiert durch die Verschreibung von Antibiotika innerhalb von 7 Tagen nach Diagnose. Die analysierten Risikofaktoren beinhalteten Alter, Geschlecht und chronische Begleiterkrankungen.

In die Analyse gingen die Daten von 1.837.080 Versicherten ein. Unter diesen identifizierten die Autoren für den Studienzeitraum 35.011 Fälle einer CAP bei 32.007 Patienten, was eine Inzidenzrate von 9,7 pro 1000 Personenjahren er- gab. Bei 2418 Patienten (7,6\%) kam es zu mehr als einer CAP-Episode, die Hospitalisierungsrate betrug 46,5\%. Die 30-Tages-Mortalität lag bei 12,9\% (4516 Patienten). Hospitalisierte Patienten wiesen eine Krankenhaus-Mortalität von $17,2 \%$ und eine 30-Tages-Mortalität von $21,9 \%$ auf, bei den ambulanten Patienten lag diese bei 5,0\%. Diese zeigte eine deutliche Altersabhängigkeit und betrug bei ambulanten Patienten im Alter unter 60 Jahren nur 0,3\%. In der multivariaten Analyse zeigte sich die Inzidenz einer CAP assoziiert mit einem höheren Alter, männlichem Geschlecht und den Komorbiditäten, wobei sich die höchsten Odds Ratios (OR) für chronische neurologische (OR 2,4), Lungen- (OR 2,3) oder immunsuppressive Erkrankungen (OR 2,1) ergaben. Im Hinblick auf die 30-Tages-Mortalität blieben dieselben Prädiktoren signifikant. Das höchste Mortalitätsrisiko bestand bei chronischen neurologischen (OR 2,3) und malignen Erkrankungen (OR 2,0).

\section{FAZIT}

Die ambulant erworbene Pneumonie stellt sowohl bei ambulanten als auch bei hospitalisierten Patienten eine große Belastung im Hinblick auf Inzidenz, Morbidität und Gesamtmortalität dar. Nach Ansicht der Autoren sind daher Maßnahmen nötig, die das Bewusstsein für die Erkrankung steigern und die Behandlungsqualität verbessern, beispielsweise eine adäquate Notfallversorgung schwerer Fälle oder eine Pneumokokken-Impfung zur Prävention.

Dr. med. Johannes Weiß, Bad Kissingen 